\title{
Protective Effect of Wheat Derived Non-specific lipid-transfer Protein 2 on Vascular Endothelium Inflammation
}

\author{
Emanuela Leoncini $^{1, \#}$, Cecilia Prata ${ }^{2, \#}$, Marco Malaguti ${ }^{1}$, Cristina Angeloni $^{3}$, Luca Massaccesi $^{1}$, \\ Sara Bosi ${ }^{4}$, Valeria Bregola ${ }^{4}$, Ilaria Marotti ${ }^{4}$, Giovanni Dinelli ${ }^{4}$, Silvana Hrelia ${ }^{1{ }^{*}}$ \\ ${ }^{1}$ Department for Life Quality Studies, Alma Mater Studiorum, University of Bologna, 47921 Rimini, Italy \\ ${ }^{2}$ Department of Pharmacy and Biotechnology, Alma Mater Studiorum, University of Bologna, 40126 Bologna, Italy \\ ${ }^{3}$ School of Pharmacy, University of Camerino, Camerino, Italy \\ ${ }^{4}$ Department of Agricultural and Food Sciences, Alma Mater Studiorum, University of Bologna, 40127 Bologna, Italy \\ ${ }^{\#}$ These authors contributed equally to this work. \\ *Corresponding author: silvana.hrelia@unibo.it
}

\begin{abstract}
The important functions of the endothelium and the relationship between cardiovascular risk factors and endothelial dysfunction suggest the primary role of this tissue as a target for dietary strategies aimed at the prevention from related diseases. Cereals are key component of a healthy and balanced diet, and the presence of non-specific lipid-transfer protein 2 (nsLTP2) in wheat represents an added value to contribute to maintain the functionality of the vascular endothelium and consequently of the cardiovascular system. Indeed, nsLTP2 downregulates the expression of the main cell adhesion molecules induced by a pro-inflammatory cytokine and, meanwhile, upregulates heme oxigenase-1, exerting a cytoprotective/anti-inflammatory activity. Therefore, nsLTP2 might represent a food-derived tool to protect the vascular system against several pathological conditions.
\end{abstract}

Keywords: wheat, bioactive peptides, nsLTP2, vascular endothelium, cytoprotection, inflammation

Cite This Article: Emanuela Leoncini, Cecilia Prata, Marco Malaguti, Cristina Angeloni, Luca Massaccesi, Sara Bosi, Valeria Bregola, Ilaria Marotti, Giovanni Dinelli, and Silvana Hrelia, "Protective Effect of Wheat Derived Non-specific lipid-transfer Protein 2 on Vascular Endothelium Inflammation.” Journal of Food and Nutrition Research, vol. 6, no. 6 (2018): 386-392. doi: 10.12691/jfnr-6-6-6.

\section{Introduction}

It has been ascertained that several well-known cardiovascular risk factors, such as arterial hypertension, hypercholesterolaemia, diabetes mellitus and smoking can affect the endothelium generating the activation of endothelial cells. This leads to a deregulation of endothelium homeostatic functions, causing a shift toward reduced endothelium-dependent vasodilation, increased inflammation and a pro-thrombotic state (phenotype) that eventually results in overt cardiovascular diseases. In fact, endothelial dysfunction has been observed in the early stage of most cardiovascular diseases [1] and is associated with various pathologies including hypertension, diabetes, atherosclerosis pulmonary hypertension, ischaemic heart diseases and chronic kidney disease [2]. The severity of endothelial dysfunction has been shown to have a prognostic value for cardiovascular pathologic events and its early clinical detection may become a critical point in cardiovascular disease prevention. In the cardiovascular continuum, endothelial activation/dysfunction represent reversible conditions, which can be targeted by nutritional strategies aimed at cardiovascular diseases contention. Although being a complex and multifactorial phenomenon, endothelial dysfunction is tightly associated with an increased production of reactive oxygen species (ROS) [3] leading to oxidative stress which is responsible for an increased vascular endothelial permeability and coupled with alterations in endothelial signal transduction and redox-regulated transcription factors [4,5].

The susceptibility of vascular cells to oxidative stress is a function of the overall balance between the degree of oxidative stress and cell antioxidant defence capability [6]. Once defined as an imbalance between pro-and antioxidants, oxidative stress is now regarded as an impaired redox signalling and equilibrium, ultimately leading to cellular damage [2]. Another common underlying mechanism of endothelial dysfunction is represented by inflammation, which is closely interrelated with oxidative stress [7]. Inflammation might turn from a physiological defence response to a chronic condition, in which a disruption of the normal function of endothelial cells takes place. Accordingly, there is a persistent low-grade inflammatory phenotype of the vasculature observed in most cardiovascular diseases [8]. Various inflammatory cytokines are involved in this process and, among them, Tumor necrosis factor-alpha (TNF- $\alpha$ ) is one of the major molecular mediators of chronic inflammation [9]. For example, circulating levels of $\mathrm{TNF} \alpha$ are elevated in patients suffering severe heart failure [10] and these levels have a 
directly proportional correlation with the patient's New York Heart Association (NYHA) functional class. Previous reports showed the pro-inflammatory activities of TNF- $\alpha$ in human endothelial cells [11]. These activities included the modulation of the expression of adhesion molecules such as Vascular Cell Adhesion Molecule 1 (VCAM-1) and Intercellular Adhesion Molecule 1 (ICAM-1).

Prolonged use of commercially available pharmacological therapies against chronic inflammatory diseases is often associated with various side effects. Given the concerns about the side effects, there has been also a substantial increase in public and scientific awareness about natural compounds and their derivatives as safer alternatives or coadjutant for the treatment and management of chronic diseases. A substantial amount of scientific evidence has suggested that food-derived compounds can modulate chronic inflammatory conditions affecting the balance between pro/anti-inflammatory cytokines [12,13]. Dietary proteins, a source of essential amino acids, necessary for growth and maintenance of physiological functions, may also represent a source of biologically active peptides. These molecules, usually smaller that $10 \mathrm{kDa}$ [14], may exist naturally in food or be encoded within protein primary sequence, from which they can be released mainly through proteolysis (during gastrointestinal digestion in vivo, bacterial fermentation and ripening in food processing or in vitro enzymatic hydrolysis) in order to be bioactive [15,16]. Depending on the aminoacidic composition of the polypeptide chain these peptides can exhibit a wide range of biological activities (antimicrobial properties, blood pressure-lowering capacity, ACE - inhibitory effects, cholesterol-lowering ability, antithrombotic and antioxidant activities, mineral binding and opioid-like activities), which can be relevant in health promotion against various chronic and degenerative disorders such as CVD [17].

In the last decade, the presence of the bioactive peptide lunasin has been reported in cereals [18], but we demonstrated the absence of lunasin in soft and durum wheat kernels by chemical and molecular analyses [19]. On the other hand, the analysis of different wheat extracts purified by chromatographic techniques allowed the identification of non-specific lipid-transfer protein (nsLTP) type 2 from Triticum turgidum subsp. turanicum [20] with gastro and intestinal resistance. Moreover, in human umbilical vein endothelial cells (HUVEC) nsLTP2 has been demonstrated to exert antioxidant activity and potential cytoprotective effects following oxidative or inflammatory stimulations [20].

In this paper, using a cell model of endothelial dysfunction (HUVECs challenged with TNF- $\alpha$ ), we investigated the potential ability of wheat derived nsLTP2 to modulate not only vascular adhesion molecules, but also Heme oxygenase (HO)-1, the inducible isoform of the first and rate-limiting enzyme of heme degradation, that has been recognized to have major immunomodulatory and anti-inflammatory properties [21]. We demonstrated that wheat-derived nsLTP2 may contribute to vascular endothelium health protection by modulating specific markers of inflammatory processes, such as adhesion molecules and cellular regulators of inflammation as HO-1, thus representing a potential tool to protect the vascular system against various stressors.

\section{Materials and Methods}

\subsection{Materials}

Medium M200 phenol red free, LGS supplement kit \#S-003-K and HUVECs (Human umbilical endothelial vein cells) were purchased from GIBCO (Thermo-Fischer Scientific, Waltham, MA USA). Recombinant Human TNF- $\alpha$ was purchased from PeproTech (PeproTech House, London UK). Fetal bovine serum (FBS), bovine serum albumin (BSA), Glycine for electrophoresis, Trizma ${ }^{\circledR}$ base, gelatin from bovine skin, Dimethyl sulfoxide (DMSO), Phosphate buffered saline (PBS), mammalian protease inhibitor mixture \#P8340, Sodium Fluoride (NaF), Phenylmethanesulfonyl fluoride (PMSF), Sodium pyrophosphate dibasic, Sodium orthovanadate, sodium pyruvate, Zinc protoporphyrin IX (ZnPPIX) and Tin Protoporphyrin IX (SnPPIX) and Anti- $\beta$ actin antibody (mouse) were purchased from Sigma-Aldrich (St. Louis, MO, USA). Nitrocellulose membrane was from GEHealthcare. Mini protean ${ }^{\circledR}$ TGX Gels, Clarity ${ }^{\mathrm{TM}}$ Western ECL Blotting Substrate, DC protein assay kit and Precision Plus Protein ${ }^{\mathrm{TM}}$ All Blue Prestained Protein Standards were purchased from BIORAD (Hercules, CA, USA). Anti HO-1 antibody (rabbit), Anti VCAM-1 antibody (rabbit), HRP-linked Antibody Anti-mouse IgG, HRP-linked Antibody Anti-rabbit were from Cell Signalling (Cell Signaling Technology, Leiden The Netherlands). Anti-ICAM-1 antibody (rabbit) was obtained from Abcam (Cambridge, MA USA). Purified peptide nsLTP2 was provided by Institute of Food Research - Norwich - UK within the European FP7 programme Bacchus and has been fully characterized as reported in [19].

\subsection{Methods}

\subsubsection{Cells Culture and Treatments}

Human Umbilical vein endothelial cells (HUVECs) were grown in standard conditions (humidified atmosphere at $37^{\circ} \mathrm{C}$ and $5 \% \mathrm{CO}_{2}$ ) in Phenol red free M200 medium (Thermo-Fischer Scientific) supplemented with $10 \%$ decomplemented fetal bovine serum (FBS) (SigmaAldrich), L-glutamine, growth factors (LSSG Kit Thermo-Fischer Scientific) which was changed every $48 \mathrm{~h}$, as previously reported [22].

Cells were cultured until they reached $80 \%$ confluence and successively seeded into gelatine-coated multiwell plates for treatments. Experiments were conducted with cells between passages 3-7. HUVECs were treated or not with nsLTP2 at final concentration of $0.01 \mu \mathrm{M}$ for $24 \mathrm{~h}$. Afterwards, inflammatory stress was induced using the inflammatory cytokine TNF- $\alpha(20 \mathrm{ng} / \mathrm{mL})$. The $10 \mathrm{nM}$ concentration of nsLTP2 was chosen because it has been previously demonstrated to exert antioxidant and cytoprotective effects in HUVECs following oxidative and inflammatory stimulation [20].

\subsubsection{PAGE and Immunoblotting}

HUVECs $\left(2 \times 10^{5}\right)$, seeded in 6-well plates were pre-treated for $24 \mathrm{~h}$ with $0.01 \mu \mathrm{M}$ nsLTP2 and further $24 \mathrm{~h}$ 
in the presence or absence of $20 \mathrm{ng} / \mathrm{mL}$ TNF- $\alpha$. At the end of the treatment cells were washed twice with ice-cold PBS and lysed in Radioimmunoprecipitation assay (RIPA) buffer supplemented with protease inhibitors mixture (Sigma) and phosphatase inhibitors. Cells were detached from the plastic support using cell-scraper, left on ice to solubilize for 15 min with periodic vortexing and subsequently centrifuged at $12000 \mathrm{rpm}$ for $15 \mathrm{~min}$ to separate the supernatants. Protein content was determined by DC protein assay kit, (Bio-Rad), using Bovine Serum Albumin (BSA) solubilized in RIPA buffer as a standard.

Samples $(10 \mu \mathrm{g} /$ lane $)$ were loaded on a $10 \%$ SDS-PAGE Mini-Protean ${ }^{\circledR}$ TGX ${ }^{\mathrm{TM}}$ (Bio-Rad Laboratories) and electrophoresed at $200 \mathrm{~V}$ for 30-40 min. Samples were loaded alongside $5 \mu \mathrm{L}$ of Precision Plus Protein ${ }^{\mathrm{TM}}$ All Blue Prestained Protein Standards (Bio-Rad) to enable identification of proteins of interest. The separated proteins were then blotted on a nitrocellulose membrane (Hybond-C; GE Healthcare, Buckinghamshire, UK) by applying to the electrophoretic cell, an electric field of $110 \mathrm{~V}$ for $90 \mathrm{~min}$. Non-specific binding to membrane was blocked with Tris-buffered saline/Tween (TTBS), containing $5 \%$ non-fat dried milk or BSA for 1 hour at room temperature. Blots were probed with primary antibodies overnight at $4{ }^{\circ} \mathrm{C}$ with gentle shaking, washed with TTBS ( $3 \times 5 \mathrm{~min}$ ) and then incubated for $1 \mathrm{~h}$ at room temperature with secondary horseradish peroxidase (HRP) conjugates antibodies.

Protein bands were detected by chemiluminescence, using Clarity ${ }^{\mathrm{TM}}$ Western ECL Blotting Substrate (Bio-Rad) according to manufacturer's protocol. Densitometric analysis of specific immunolabeled bands was performed using the Chemidoc MP (Bio-Rad, Hercules, CA) software. Band intensity was normalized to the intensity of the corresponding $\beta$-actin band and results were expressed as fold increase relative to control cells or TNF- $\alpha$ treated cells.

\subsubsection{Fluorescence Immunostaining}

HUVECs were seeded on glass cover-slips and treated with $10 \mathrm{nM}$ nsLTP2 for $24 \mathrm{~h}$. At the end of the treatment, cells were washed with PBS, fixed with 3\% paraformaldehyde for $15 \mathrm{~min}$, washed with $0.1 \mathrm{M}$ glycine in PBS and permeabilized in $70 \%$ ice cold ethanol. After fixing, the cells were incubated overnight at $4{ }^{\circ} \mathrm{C}$ with anti HO-1 primary antibody. Subsequently the samples were washed with $1 \%$ BSA in PBS and incubated with DyLight 488 labeled secondary antibody (KPL, Gaithersburg, MD) for $1 \mathrm{~h}$ at room temperature. Nuclei were labelled with DAPI $(0.01 \mu \mathrm{g} / \mathrm{mL}$ final concentration) for $10 \mathrm{~min}$ at room temperature. Preparations were mounted on microscope slides with Vectashild (Vector Laboratories, Burlingame, CA) and images were acquired with Axio Scope.A1 microscope (Zeiss) at 100x magnification.

\subsubsection{Statistical Analysis}

Data represent mean values with \pm respective standard deviation (SD) corresponding to three or more independent experiments. Differences between means were analysed by unpaired Student's t-test with $\mathrm{P}<0.05$ considered to be significant.

\section{Results}

\subsection{Wheat nsLTP2 Exerts Anti-inflammatory Effect Modulating Adhesion Molecules Expression}

The endothelial expression of cell-surface adhesion molecules, such as VCAM-1, ICAM-1 represents a marker of endothelial activation, which is typically induced by pro-inflammatory cytokines.

In order to assess nsLTP2 potential anti-inflammatory activity, experiments were conducted in HUVECs pre-treated with $10 \mathrm{nM}$ nsLTP2 for $24 \mathrm{~h}$ before the inflammatory stimulation by TNF- $\alpha$. Immunoblotting analysis showed that $24 \mathrm{~h}$ treatment with $20 \mathrm{ng} / \mathrm{mL}$ TNF- $\alpha$ caused a significant increase in the expression of VCAM-1 and ICAM-1 which is significantly decreased after a 24 h pre-treatment with $10 \mathrm{nM}$ nsLTP2 (Figure 1).

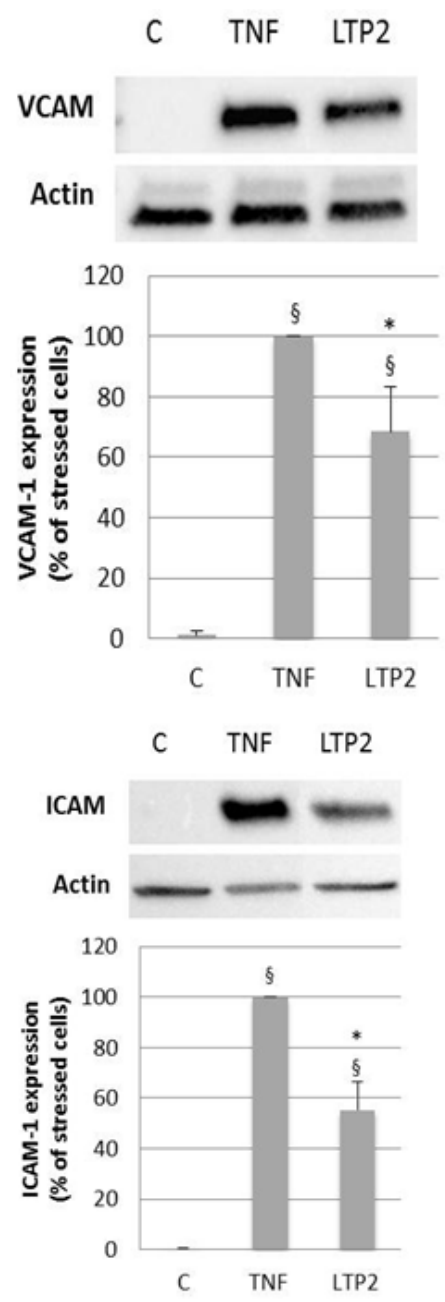

Figure 1. Effect of nsLTP2 on cell adhesion molecule expression in HUVECs treated with TNF- $\alpha$. After $10 \mathrm{nM}$ nsLTP2 (LTP2) pre-treatment for $24 \mathrm{~h}$, HUVECs were stressed with $20 \mathrm{ng} / \mathrm{mL}$ TNF- $\alpha$. Cell lysates were subjected to SDS-PAGE and Western blotting analysis, using primary antibodies directed to VCAM-1, ICAM-1. Images show a representative experiment of three independent analyses. Densitometric analysis of bands intensity, normalized to actin, was expressed as \% of stressed cells and was carried out with the ChemiDoc MP System (BIORAD). Data represent means \pm SD of three independent experiments. ${ }^{\S} \mathrm{p}<0.05$, vs control HUVECs; ${ }^{*} \mathrm{p}<0.05$, vs HUVECs stressed with TNF- $\alpha$ 


\subsection{Wheat nsLTP2 Effect on Heme Oxygenase (HO-1) in Vascular Endothelial Cells}

Since HO-1 is usually involved in inflammatory processes, in order to verify if wheat peptide nsLTP2 affect HO-1 expression, HUVECs were treated with 10 $\mathrm{nM}$ nsLTP2 for $24 \mathrm{~h}$ and cell lysates were subjected to immunoblotting analysis. HUVECs treatment with $10 \mathrm{nM}$ nsLTP2 for $24 \mathrm{~h}$ caused a significant increase in HO-1 protein expression, with respect to control cells (Figure 2).

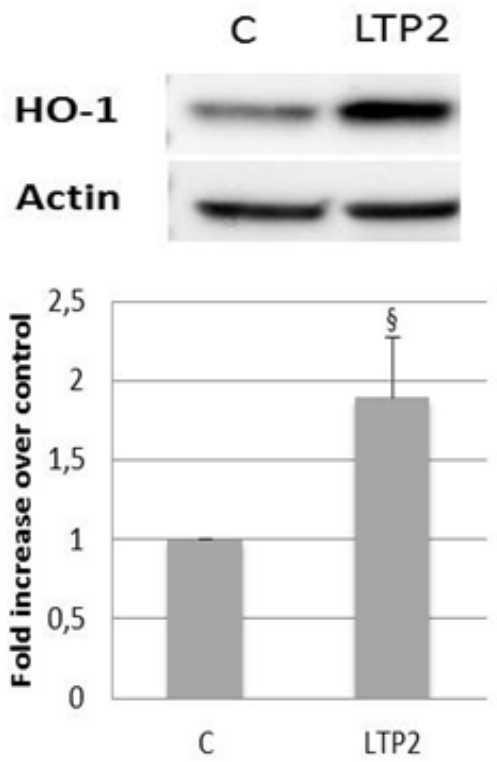

Figure 2. Effect of nsLTP2 on HO-1 protein expression level in HUVECs. After treatment with $10 \mathrm{nM}$ nsLTP2 for $24 \mathrm{~h}$, cells lysates were subjected to SDS-PAGE and Western blotting analysis of HO-1 protein expression levels. The image shows a representative experiment of three independent analyses. Densitometric analysis of HO-1 expression, normalized to actin, is expressed as fold increase over control and was carried out with the ChemiDoc MP System (BIORAD). Data represent means $\pm \mathrm{SD}$ of three independent experiments. §p $<0.05$, vs control HUVECs

The fluorescent immunostaining analysis, performed in the same experimental conditions, revealed that in HUVECs treated with $10 \mathrm{nM}$ nsLTP2 for $24 \mathrm{~h}$ a strong increase in cell fluorescence was detectable (Figure 3), supporting the previously reported result.

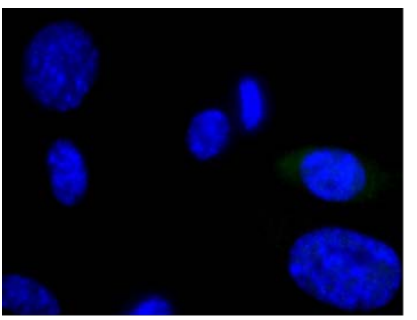

Control

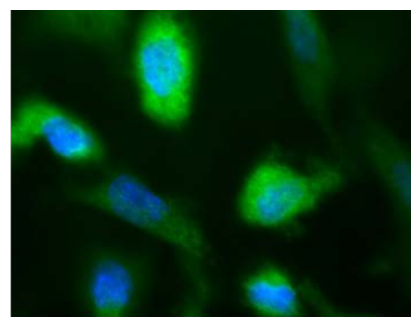

nsLTP2
Figure 3. Immunofluorescence staining of HO-1 in HUVECs after 10 nM nsLTP2 treatment for $\mathbf{2 4}$ h After treatment with $10 \mathrm{nM}$ nsLTP2, HUVECs were immunolabeled with anti-HO-1 antibody and relative fluorescent FITCH-conjugated secondary antibody then visualized using immunofluorescence microscopy (Zeiss Axio Scope.A1, magnification 100x). Nuclei were stained with DAPI

\subsection{Comparison of the Inducing Capacity of nsLTP2 and Statins on HO-1 in Endothelial Cells}

As statins, beside their HMG-CoA reductase antagonist effect, exert cytoprotective/anti-inflammatory activity by inducing HO-1 expression, a comparison between nsLTP2 and Atorvastatin and Simvastatin effect was performed. Immunoblotting analysis showed that upon HUVECs treatment with both statins and nsLTP2 at the same concentration (10 nM) for $24 \mathrm{~h}, \mathrm{HO}-1$ expression was significantly up regulated (Figure 4).

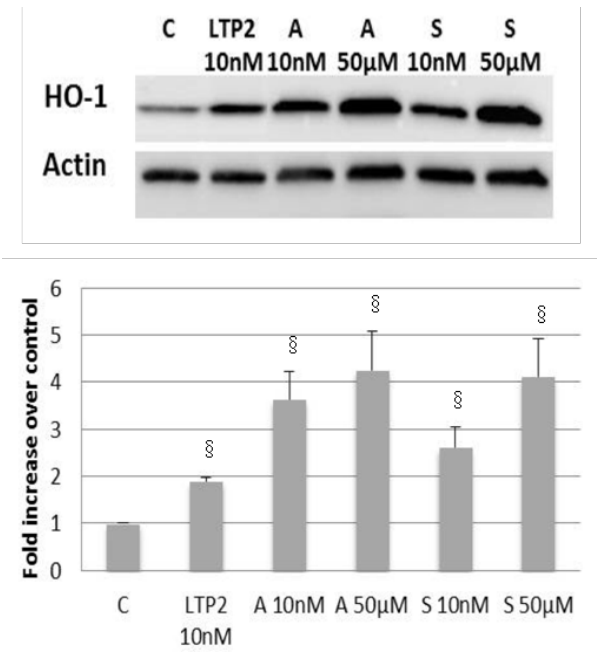

Figure 4. Effect of nsLTP2 (LTP2), Atorvastatin and Simvastatin on HO-1 protein expression level. HUVECs were treated with $10 \mathrm{nM}$ nsLTP2 (LTP2), $10 \mathrm{nM}-50 \mu \mathrm{M}$ Atorvastatin (A) or $10 \mathrm{nM}-50 \mu \mathrm{M}$ Simvastatin (S) for $24 \mathrm{~h}$. Cell lysates were subjected to SDS-PAGE and Western blotting analysis of HO-1 protein expression level. Densitometric analysis of HO-1 expression, normalized to actin, is expressed as fold increase over control and was carried out with the ChemiDoc MP System (BIORAD). Data represent means \pm SD of three independent experiments. §p $<0.05$, compared with control HUVECs

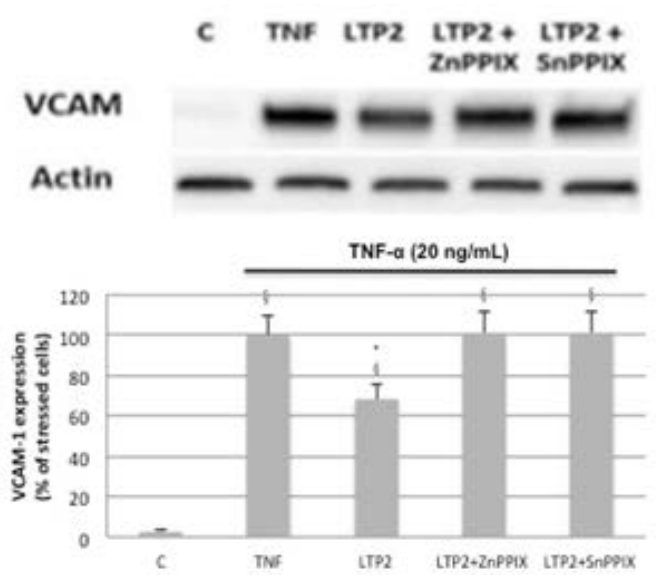

Figure 5. Effect of nsLTP2 on VCAM-1 expression in HUVECs treated with TNF- $\alpha$ in the presence or absence of HO-1 inhibitors. HUVECs were treated with $10 \mathrm{nM}$ nsLTP in the presence or absence of HO-1 inhibitors (10nM ZnPP IX and SnPP IX) and subsequently treated with $20 \mathrm{ng} / \mathrm{mL}$ TNF- $\alpha$. Cell lysates were subjected to SDS-PAGE and Western blotting analysis of VCAM protein expression level. Densitometric analysis of VCAM expression normalized to actin is expressed as \% of stressed cells and was carried out with the ChemiDoc MP System (BIORAD). Data represent means \pm SD of three independent experiments. ${ }^{\S} \mathrm{p}<0.05$, compared with control HUVECs, ${ }^{*} \mathrm{p}<0.05$, compared with HUVECs stressed by TNF- $\alpha$. 
In order to clarify if the observed modulatory effect exerted by nsLTP2 on adhesion molecules could be mediated by the induction of HO-1, HUVECs were treated with two HO-1 inhibitors: Zinc protoporphyrin IX (ZnPPIX) and Tin Protoporphyrin IX (SnPPIX) for 24h.

In HUVECs pre-treated with $10 \mathrm{nM}$ nsLTP2 for $24 \mathrm{~h}$ a decrease in the expression of VCAM-1 induced by TNF- $\alpha$ was detectable, while this effect was abrogated when cells were treated with both ZnPPIX and SnPPIX, suggesting that the induced expression of HO-1 may be involved in the modulatory effect of nsLTP2 on adhesion molecules (Figure 5).

\section{Discussion}

The main cardiovascular risk factors are associated with a condition of oxidative stress and inflammation able to induce a "dysfunctional" endothelial state. The key role of the endothelium in vascular homeostasis maintenance and the observed correlation between endothelial dysfunction and cardiovascular diseases onset and development make this tissue a primary target for dietary strategies aimed at cardiovascular diseases prevention. A direct correlation between the intake of whole grains, including wheat and derived products and the reduction in risk of developing chronic degenerative diseases has been reported [23,24]. Initially attributed to the abundance of fibers and micronutrients, this beneficial effect is today more centered on their bioactive components content, responsible for the nutraceutical value of these foods [25]. With the aim of searching for peptides, which may characterize different wheat varieties for their biological activity and nutraceutical properties, we previously identified a $7 \mathrm{kDa}$ peptide (non-specific lipid-transfer protein type 2, nsLTP2) from a pool of soft and durum wheat varieties and from KAMUT ${ }^{\circledR}$ khorasan wheat (Triticum turgidum ssp. turanicum) [19]. In a previous study we demonstrated the antioxidant/cytoprotective activity of nsLTP2 in the same experimental model [20]. Since oxidative stress and inflammation represent the basis of endothelial dysfunction, these activities could be considered as potentially beneficial for vascular health maintenance.

The aim of the present work is was to deepen the analysis of the mechanism at the basis of potential anti-inflammatory activity of nsLTP2. Experiments were conducted in order to verify a possible modulatory effect exerted by nsLTP2 on adhesion molecule expression, since they are overexpressed in inflammatory conditions, representing a biomarker of endothelial activation. Immunoblotting analyses suggest that nsLTP2 at physiologically achievable concentration (10 $\mathrm{nM})$ is able to significantly reduce the increased expression of cell adhesion molecules observed following HUVECs treatment with TNF- $\alpha(20 \mathrm{ng} / \mathrm{ml})$. These results are in accordance with other studies in which the potential protective effects of phytochemicals was analysed in the same experimental model of endothelial inflammation $[26,27,28,29]$. The analysis of the potential anti-inflammatory effect of nsLTP2 was extended to the evaluation of its effect on HO-1 expression. HO-1, beside its well known role in heme catabolism, has been recognized as being part of endogenous antioxidant defense system [30]. Numerous scientific evidence highlighted the important physiological and beneficial role of HO-1 in the vasculature particularly on vascular endothelium toward which it exerts pleiotropic, including antiapoptotic, anti-inflammatory and antioxidant actions [31]. Overexpression of HO-1 in human endothelial cells has been shown to protect against tert-butyl hydroperoxide cytotoxicity [32], pro-apoptotic cytokines [33] and crotonaldhyeide [34]. In addiction, HO-1 decreases oxidative stress induced by $\mathrm{H}_{2} \mathrm{O}_{2}$ and/or NADPH oxidase [35] and may attenuate inflammatory response induced by LPS, TNF- $\alpha$ and other inflammatory cytokines. OH-1 has been demonstrated to decrease the up-regulation of cyclo-oxygenase-2 (COX-2), E-selectin, vascular cell adhesion molecule-1 (VCAM-1), intercellular adhesion molecule-1 (ICAM-1), and monocyte chemoattractant protein-1 (MCP-1) secretion [31].

Therefore, in order to verify if nsLTP2 modulates HO-1 expression, HUVECs were treated with $10 \mathrm{nM}$ nsLTP2 for $24 \mathrm{~h}$ and cell lysates were subjected to immunoblotting analysis. HUVECs treatment with nsLTP2 caused a significant increase in HO-1 protein expression, compared to control cells. This data suggest an anti-inflammatory activity exerted by the selected peptide due to the activation of one of the enzyme linked to endogenous antioxidant defence system. These results are confirmed by immunofluorescent analysis.

Some common dietary phytochemicals are known HO-1 inducers [36] as well as drugs currently used in therapy [31] for example, statins. In particular, simvastatin, rosuvastatin and atorvastatin have been shown to increase HO-1 promoter activity and increase the antioxidant capacity in endothelial cells [37], displaying a vasculoprotective and anti-inflammatory activity independent from their antagonist effect on HMG-CoA reductase. Therefore, nsLTP2 effect on HO-1 expression was compared to that of statins. HUVECs were treated with simvastatin, atorvastatin or nsLTP2 for $24 \mathrm{~h}$ and HO-1 protein expression was examined by immunoblotting. HO-1 up-regulation occurred following cell treatment with both nsLTP2 and statins at the same concentration (10nM), this up regulation increases at higher statin concentrations (50 $\mu \mathrm{M})$, commonly used to evaluate the effect of these drugs on biological activities, as reported in literature [38]. Since the induction of HO-1 has been linked to the inhibition of endothelial adhesion molecules expression and this mechanism is considered to be, at least in part, responsible for the resulting amelioration of inflammation at vascular level [39], we next examined whether the HO-1 up-regulation, induced by nsLTP2, was correlated to the down-regulation of adhesion molecule expression (i.e. VCAM) induced by TNF- $\alpha$. Consequently, experiments were conducted using two HO-1 inhibitors: Zinc Protoporphyrin IX (ZnPPIX) and Tin Protoporphyrin IX (SnPPIX). HUVEC pre-treatment with nsLTP2 resulted in a decreased expression of VCAM-1 induced by TNF- $\alpha$ and this effect is counteracted by HO-1 inhibitors, suggesting that the induced expression of HO-1 may contribute to the underlying mechanism of the anti-inflammatory activity exerted nsLTP2. Similarly, this mechanism has been reported for other natural compounds [40].

Taken together, these results suggest that nsLTP2, as a HO-1 inducer, might represent a potential tool to protect the vascular system against various stressors, preventing several pathological conditions. 
These results are particularly relevant, as they were observed at physiologically achievable concentrations that were chosen according to data presented in the literature [41].

\section{Conclusions}

There are many risk factors able to induce oxidative stress and inflammation and create an endothelial dysfunctional state. The important functions of the endothelium and the relationship between risk factors and endothelial dysfunction in the onset of cardiovascular disease makes this tissue a primary target for dietary strategies aimed at the prevention of the onset of these diseases. Cereals are key component of a healthy and balanced diet, as highlighted by their location at the base of different national nutritional pyramids. Thanks also to the presence of nsLTP2, they may contribute to maintain the functionality not only of the cardiovascular system and in particular of the vascular endothelium. A randomized, double-blind, clinical trial is now in progress to evaluate haemodynamic and metabolic effects of nsLTP2-rich wheat products assumption in a cohort of pre-hypertensive subjects.

\section{Acknowledgements}

This work has been financially supported by the BACCHUS project (FP7 European Commission Grant Agreement 312090).

\section{References}

[1] Yang, Z., L. Chen, C. Su, W.H. Xia, Y. Wang, J.M. Wang, F. Chen, Y.Y. Zhang, F. Wu, S.Y. Xu, X.L. Zhang, and J. Tao, "Impaired endothelial progenitor cell activity is associated with reduced arterial elasticity in patients with essential hypertension", Clin Exp Hypertens, 32 (7), 444-52, 2010.

[2] Montezano, A.C. and R.M. Touyz, "Molecular mechanisms of hypertension--reactive oxygen species and antioxidants: a basic science update for the clinician", Can J Cardiol, 28 (3), 288-95, 2012.

[3] Munzel, T. and E. Schulz, "[Treatment of coronary heart disease with nitric oxide donors]", Pharm Unserer Zeit, 39 (5), 359-68, 2010.

[4] Lum, H. and K.A. Roebuck, "Oxidant stress and endothelial cell dysfunction", Am J Physiol Cell Physiol, 280 (4), C719-41, 2001.

[5] Hadi, H.A., C.S. Carr, and J. Al Suwaidi, "Endothelial dysfunction: cardiovascular risk factors, therapy, and outcome", Vasc Health Risk Manag, 1 (3), 183-98, 2005.

[6] Higashi, Y., K. Noma, M. Yoshizumi, and Y. Kihara, "Endothelial function and oxidative stress in cardiovascular diseases", Circ J, 73 (3), 411-8, 2009.

[7] Karbach, S., A.L. Croxford, M. Oelze, R. Schuler, D. Minwegen, J. Wegner, L. Koukes, N. Yogev, A. Nikolaev, S. Reissig, A. Ullmann, M. Knorr, M. Waldner, M.F. Neurath, H. Li, Z. Wu, C. Brochhausen, J. Scheller, S. Rose-John, C. Piotrowski, I. Bechmann, M. Radsak, P. Wild, A. Daiber, E. von Stebut, P. Wenzel, A. Waisman, and T. Munzel, "Interleukin 17 drives vascular inflammation, endothelial dysfunction, and arterial hypertension in psoriasis-like skin disease", Arterioscler Thromb Vasc Biol, 34 (12), 2658-68, 2014.

[8] Daiber, A., S. Steven, A. Weber, V.V. Shuvaev, V.R. Muzykantov, I. Laher, H. Li, S. Lamas, and T. Munzel, "Targeting vascular (endothelial) dysfunction", Br J Pharmacol, 174 (12), 1591-1619, 2017.

[9] Aggarwal, B.B., S.C. Gupta, and B. Sung, "Curcumin: an orally bioavailable blocker of TNF and other pro-inflammatory biomarkers", Br J Pharmacol, 169 (8), 1672-92, 2013.
[10] Levine, B., J. Kalman, L. Mayer, H.M. Fillit, and M. Packer, "Elevated circulating levels of tumor necrosis factor in severe chronic heart failure", N Engl J Med, 323 (4), 236-41, 1990.

[11] Steyers, C.M., 3rd and F.J. Miller, Jr., "Endothelial dysfunction in chronic inflammatory diseases", Int J Mol Sci, 15 (7), 11324-49, 2014.

[12] Kuntz, S., S. Rudloff, J. Ehl, R.G. Bretzel, and C. Kunz, "Food derived carbonyl compounds affect basal and stimulated secretion of interleukin-6 and -8 in Caco-2 cells", Eur J Nutr, 48 (8), 499-503, 2009.

[13] Chanput, W., J. Mes, R.A. Vreeburg, H.F. Savelkoul, and H.J. Wichers, "Transcription profiles of LPS-stimulated THP-1 monocytes and macrophages: a tool to study inflammation modulating effects of food-derived compounds", Food Funct, 1 (3), 254-61, 2011.

[14] Malaguti, M., G. Dinelli, E. Leoncini, V. Bregola, S. Bosi, A.F. Cicero, and S. Hrelia, "Bioactive peptides in cereals and legumes: agronomical, biochemical and clinical aspects", Int J Mol Sci, 15 (11), 21120-35, 2014.

[15] Kitts, D.D. and K. Weiler, "Bioactive proteins and peptides from food sources. Applications of bioprocesses used in isolation and recovery", Curr Pharm Des, 9 (16), 1309-23, 2003.

[16] Chakrabarti, S., F. Jahandideh, and J. Wu, "Food-derived bioactive peptides on inflammation and oxidative stress", Biomed Res Int, 2014 608979, 2014.

[17] Erdmann, K., B.W. Cheung, and H. Schroder, "The possible roles of food-derived bioactive peptides in reducing the risk of cardiovascular disease", J Nutr Biochem, 19 (10), 643-54, 2008.

[18] Nakurte, I., K. Klavins, I. Kirhnere, J. Namniece, L. Adlere, J. Matvejevs, A. Kronberga, A. Kokare, V. Strazdina, L. Legzdina, and R. Muceniece, "Discovery of lunasin peptide in triticale (X Triticosecale Wittmack)", Journal of Cereal Science, 56 510-514, 2012.

[19] Dinelli, G., V. Bregola, S. Bosi, J. Fiori, R. Gotti, E. Simonetti, C. Trozzi, E. Leoncini, C. Prata, L. Massaccesi, M. Malaguti, R. Quinn, and S. Hrelia, "Lunasin in wheat: a chemical and molecular study on its presence or absence", Food Chem, 151 520-5, 2014.

[20] Bosi, S., J. Fiori, G. Dinelli, N.M. Rigby, E. Leoncini, C. Prata, V. Bregola, I. Marotti, R. Gotti, M. Naldi, L. Massaccesi, M. Malaguti, P. Kroon, and S. Hrelia, "Isolation and characterization of wheat derived non specific Lipid Transfer Protein 2 (nsLTP2)", J Food Sci, 83 (6), 1516-1521, 2018.

[21] Paine, A., B. Eiz-Vesper, R. Blasczyk, and S. Immenschuh, "Signaling to heme oxygenase-1 and its anti-inflammatory therapeutic potential", Biochem Pharmacol, 80 (12), 1895-903, 2010.

[22] Caliceti, C., P. Rizzo, R. Ferrari, F. Fortini, G. Aquila, E. Leoncini, L. Zambonin, B. Rizzo, D. Calabria, P. Simoni, M. Mirasoli, M. Guardigli, S. Hrelia, A. Roda, and A.F.G. Cicero, "Novel role of the nutraceutical bioactive compound berberine in lectin-like OxLDL receptor 1-mediated endothelial dysfunction in comparison to lovastatin", Nutr Metab Cardiovasc Dis, 27 (6), 552-563, 2017.

[23] Slavin, J.L., D. Jacobs, L. Marquart, and K. Wiemer, "The role of whole grains in disease prevention", J Am Diet Assoc, 101 (7), 780-5, 2001.

[24] Seal, C.J. and I.A. Brownlee, "Whole-grain foods and chronic disease: evidence from epidemiological and intervention studies", Proc Nutr Soc, 74 (3), 313-9, 2015.

[25] Leoncini, E., C. Prata, M. Malaguti, I. Marotti, A. SeguraCarretero, P. Catizone, G. Dinelli, and S. Hrelia, "Phytochemical profile and nutraceutical value of old and modern common wheat cultivars", PLoS One, 7 (9), e45997, 2012.

[26] Lee, A.S., J.S. Kim, Y.J. Lee, D.G. Kang, and H.S. Lee, "AntiTNF-alpha activity of Portulaca oleracea in vascular endothelial cells", Int J Mol Sci, 13 (5), 5628-44, 2012.

[27] Xia, F., C. Wang, Y. Jin, Q. Liu, Q. Meng, K. Liu, and H. Sun, "Luteolin protects HUVECs from TNF-alpha-induced oxidative stress and inflammation via its effects on the Nox4/ROS-NFkappaB and MAPK pathways", J Atheroscler Thromb, 21 (8), 768-83, 2014.

[28] Chen, T.L., G.L. Zhu, J.A. Wang, G.D. Zhang, H.F. Liu, J.R. Chen, Y. Wang, and X.L. He, "Protective effects of isorhamnetin on apoptosis and inflammation in TNF-alpha-induced HUVECs injury", Int J Clin Exp Pathol, 8 (3), 2311-20, 2015. 
[29] Kim, D.H., S.M. Lee, Y.J. Lee, J.J. Yoon, R. Tan, Y.C. Yu, D.G. Kang, and H.S. Lee, "Effect of Paeotang on tumor necrosis factor alpha-induced vascular inflammation in human umbilical vein endothelial cells", Chin J Integr Med, 2017.

[30] Talalay, P., "Chemoprotection against cancer by induction of phase 2 enzymes", Biofactors, 12 (1-4), 5-11, 2000.

[31] Calay, D. and J.C. Mason, "The multifunctional role and therapeutic potential of $\mathrm{HO}-1$ in the vascular endothelium", Antioxid Redox Signal, 20 (11), 1789-809, 2014.

[32] Ishikado, A., Y. Nishio, K. Morino, S. Ugi, H. Kondo, T. Makino, A. Kashiwagi, and H. Maegawa, "Low concentration of 4-hydroxy hexenal increases heme oxygenase-1 expression through activation of Nrf2 and antioxidative activity in vascular endothelial cells", Biochem Biophys Res Commun, 402 (1), 99-104, 2010.

[33] Liu, X.M., K.J. Peyton, A.R. Shebib, H. Wang, R.J. Korthuis, and W. Durante, "Activation of AMPK stimulates heme oxygenase-1 gene expression and human endothelial cell survival", $A m \mathrm{~J}$ Physiol Heart Circ Physiol, 300 (1), H84-93, 2011.

[34] Lee, S.E., S.I. Jeong, G.D. Kim, H. Yang, C.S. Park, Y.H. Jin, and Y.S. Park, "Upregulation of heme oxygenase-1 as an adaptive mechanism for protection against crotonaldehyde in human umbilical vein endothelial cells", Toxicol Lett, 201 (3), 240-8, 2011.

[35] Wu, C.C., M.C. Hsu, C.W. Hsieh, J.B. Lin, P.H. Lai, and B.S Wung, "Upregulation of heme oxygenase-1 by Epigallocatechin- 3-gallate via the phosphatidylinositol 3-kinase/Akt and ERK pathways", Life Sci, 78 (25), 2889-97, 2006.

[36] Haines, D.D., I. Lekli, P. Teissier, I. Bak, and A. Tosaki, "Role of haeme oxygenase-1 in resolution of oxidative stress-related pathologies: focus on cardiovascular, lung, neurological and kidney disorders", Acta Physiol (Oxf), 204 (4), 487-501, 2012.

[37] Mrad, M.F., C.A. Mouawad, M. Al-Hariri, A.A. Eid, J. Alam, and A. Habib, "Statins modulate transcriptional activity of hemeoxygenase-1 promoter in NIH 3T3 Cells", J Cell Biochem, 113 (11), 3466-75.

[38] Yang, J.C., F. Huang, C.J. Wu, Y.C. Chen, T.H. Lu, and C.H. Hsieh, "Simvastatin reduces VCAM-1 expression in human umbilical vein endothelial cells exposed to lipopolysaccharide", Inflamm Res, 61 (5), 485-91, 2012.

[39] Olszanecki, R., A. Gebska, and R. Korbut, "The role of haem oxygenase- 1 in the decrease of endothelial intercellular adhesion molecule-1 expression by curcumin", Basic Clin Pharmacol Toxicol, 101 (6), 411-5, 2007.

[40] Li, B., Y.J. Lee, Y.C. Kim, J.J. Yoon, S.M. Lee, Y.P. Lee, D.G. Kang, and H.S. Lee, "Sauchinone from Saururus chinensis protects vascular inflammation by heme oxygenase-1 induction in human umbilical vein endothelial cells", Phytomedicine, 21 (2), 101-8, 2014.

[41] Dia, V.P., S. Torres, B.O. De Lumen, J.W. Erdman, Jr., and E.G. De Mejia, "Presence of lunasin in plasma of men after soy protein consumption", J Agric Food Chem, 57 (4), 1260-6, 2009. 